\title{
Longitudinal Stability Criteria for a Propeller-Driven Aircraft
}

\author{
Gil Iosilevskii \\ Faculty of Aerospace Engineering, Technion, Haifa 32000, Israel \\ Correspondence should be addressed to Gil Iosilevskii, igil@aerodyne.technion.ac.il
}

Received 1 September 2010; Accepted 30 November 2010

Academic Editor: Isaac Kaminer

Copyright () 2010 Gil Iosilevskii. This is an open access article distributed under the Creative Commons Attribution License, which permits unrestricted use, distribution, and reproduction in any medium, provided the original work is properly cited.

The Routh criterion is used to assess longitudinal dynamic stability of a propeller-driven aircraft. Under a few plausible assumptions on possible ranges of the pertinent stability derivatives, it reduces to a pair of simple conditions imposing a traditional aft limit (the forward of the maneuver and the neutral-speed-stability points) on the center-of-gravity position and an upper limit on the longitudinal moment of inertia. It is demonstrated that most aircraft have sufficiently small inertia to remain stable as long as their center-of-gravity is properly placed. At the same time, sailplane-like aircraft (as, e.g., long endurance UAVs), with an engine installed at the rear extremity of the aircraft, may have sufficiently high inertia to become unstable regardless of their center-of-gravity placement.

\section{Introduction}

Linear analysis of the longitudinal dynamics of a rigid aircraft can be found practically in any textbook on flight mechanics. In a nutshell, it can be recapitulated as follows. Assuming constant-density atmosphere, flat nonrotating Earth, zero thrust angle, and no control inputs, the equations governing small perturbations $\mathbf{x}=[u, \alpha, q, \theta]^{\mathrm{T}}$ of airspeed, angle-ofattack, pitch rate and pitch angle from their respective values in a trimmed straight-and-almost-level flight can be written as

$$
\mathbf{B} \cdot \dot{\mathbf{x}}=\mathbf{A} \cdot \mathbf{x},
$$

with

$$
\mathbf{A}=\left[\begin{array}{cccc}
C_{\mathrm{m}, u} & C_{\mathrm{m}, \alpha} & C_{\mathrm{m}, q} & 0 \\
C_{\mathrm{L}, u}+2 \mathrm{C}_{\mathrm{L}} & C_{\mathrm{D}}+C_{\mathrm{L}, \alpha} & C_{\mathrm{L}, q}-2 \mu & \gamma C_{\mathrm{L}} \\
C_{\mathrm{T}, u}-C_{\mathrm{D}, u}+2 \gamma C_{\mathrm{L}} & C_{\mathrm{L}}-\mathrm{C}_{\mathrm{D}, \alpha} & 0 & -C_{\mathrm{L}} \\
0 & 0 & 1 & 0
\end{array}\right]
$$

$$
\mathbf{B}=\left[\begin{array}{cccc}
0 & -C_{\mathrm{m}, \dot{\alpha}} & i_{y} & 0 \\
0 & -\left(2 \mu+C_{\mathrm{L}, \dot{\alpha}}\right) & 0 & 0 \\
2 \mu & 0 & 0 & 0 \\
0 & 0 & 0 & 1
\end{array}\right]
$$

see equation $(5.13,16)$ in $[1]$. The notation here is the same as in [1] with three exceptions: (i) an overdot replaces " $D$ " to designate a derivative with respect to reduced time; (ii) the subscript " $\mathrm{e}$ " is omitted from unperturbed (equilibrium) values of the flight-path angle and the lift and drag coefficients; (iii) " $i_{y}$ " and " $u$ " replace " $\hat{I}_{y}$ " and " $\Delta \hat{V}$ " for the reduced moment of inertia and the reduced velocity.

Setting $\mathbf{x}=\mathbf{x}_{0} \mathrm{e}^{s t}$ in (1), where $t$ is the (reduced) time and $\mathbf{x}_{0}$ and $s$ are obvious time-independent quantities, leads to the generalized eigenvalue problem $(s \mathbf{B}-\mathbf{A}) \cdot \mathbf{x}_{0}=0$, and, in turn, to the fourth order characteristic equation,

$$
a s^{4}+b s^{3}+c s^{2}+d s+e=0 .
$$


The coefficients in (4) can be identified with products of the elements of the matrices $\mathbf{A}$ and $\mathbf{B}$, but except for

$$
a=2 i_{y} \mu\left(2 \mu+C_{\mathrm{L}, \dot{\alpha}}\right),
$$

all other coefficients are rather unwieldy (Appendix A). Of course, the aircraft is stable if all the roots of (4) are in the left half-plane.

Equation (4) has no analytical solution for $s$, and therefore it has to be solved either approximately [2] or numerically. Each approach has its virtues, but in many cases the roots of (4) are not really needed-what is needed is the knowledge whether the aircraft is stable or not. In principle, stability of a system (the aircraft, in this case) can be assessed without actually solving its characteristic equation-by the Routh criterion, the number of roots of (4) which are in the right-half-plane equals the number of sign alternations in the five-member sequence $S=\{a, b, f, g, e\}$, where $f=c-a d / b$ and $g=d-b e / f$ [3, pages 253-255]. In practice, however, this approach is hardly ever used with (4) - the unwieldiness of the expressions for the members of $S$ renders it rather unattractive as compared with the instant numerical solution of the generalized eigenvalue problem leading to (4).

Notwithstanding the majority approach, we suggest that for a propeller-driven aircraft, an application of the Routh criterion to (4) yields a pair of very simple conditions with immediate design implications. One of these conditions sets a traditional aft limit (the forward of the maneuver and the neutral-speed-stability points) on the center-of-gravity position; the other sets an upper limit on the longitudinal moment of inertia. The details follow.

\section{Approximate Stability Criterion}

As a first step, we replace the moment derivatives $C_{\mathrm{m},} \bullet$ in (2) and (3) (or, to the same end, in (A.1)-(A.5) of Appendix A), all referred to the center of gravity, with combinations

$$
C_{\mathrm{m}, \bullet}=C_{\mathrm{M}, \bullet}-C_{\mathrm{L}, \bullet}\left(x_{\mathrm{cg}}-x_{\mathrm{n}}\right)
$$

of their neutral-point-referred counterparts, $C_{\mathrm{M}, \bullet}$, and lift coefficient derivatives, $C_{\mathrm{L},}$. Here, $x_{\mathrm{cg}}$ and $x_{\mathrm{n}}$ are the centerof-gravity and neutral-point positions, and “•” stands for " $\alpha$," " $\dot{\alpha}$," " $q$," and " $u$." Among the neutral-point-referred moment derivatives,

$$
\begin{gathered}
C_{\mathrm{M}, \alpha}=0, \\
C_{\mathrm{M}, q}=2 \mu\left(x_{\mathrm{m}}-x_{\mathrm{n}}\right), \\
C_{\mathrm{M}, u}=-2 C_{\mathrm{L}}\left(x_{\mathrm{s}}-x_{\mathrm{n}}\right),
\end{gathered}
$$

by definitions of the neutral $\left(x_{\mathrm{n}}\right)$, maneuver $\left(x_{\mathrm{m}}\right)$, and neutral-speed-stability $\left(x_{\mathrm{s}}\right)$ points; the last pair follows equations $(6.10,8)$ and $(6.4,26)$ of $[1]$ by $(6)$.

These substitutions do simplify the expressions for $b, \ldots, e$, but essentially do not reduce the number of terms comprising them. Hence, as a next step, we sort these terms by their magnitudes and retain a few dominant ones only. For a typical subsonic aircraft in an almost level flight, invariably

$C_{\mathrm{D}}, C_{\mathrm{D}, u}, C_{\mathrm{D}, \alpha}, C_{\mathrm{T}, u}, C_{\mathrm{L}, u}, C_{\mathrm{M}, u}, \gamma$ are of the order $10^{-1}$,

$C_{\mathrm{L}}$ is of the order $10^{0}$,

$C_{\mathrm{L}, \alpha}, C_{\mathrm{L}, \dot{\alpha}}, C_{\mathrm{L}, q}, C_{\mathrm{M}, \dot{\alpha}}$ and $C_{\mathrm{M}, q}$ are of the order $10^{1}$,

$\mu$ is of the order $10^{2}$,

$i_{y}$ is of the order $10^{2}$ or $10^{3}$.

The word "order" can be understood here (and below) in its intuitive sense; nevertheless, we shall remove possible ambiguity and define the order $O(a)$ of a quantity $a$ as $10^{\text {round }\left(\log _{10}|a|\right)}$. Thus, 4 and 30 are both of the order $10^{1}$.

Sorting out the terms in (A.2)-(A.5) by (10), and discarding all those that are of the order $10^{-2}$ (or smaller) as compared with the leading ones, leaves

$$
\begin{aligned}
& b=2 \mu\left(i_{y}\left(C_{\mathrm{L}, \alpha}+O\left(10^{-1}\right)\right)-\left(2 \mu-C_{\mathrm{L}, q}\right)\left(C_{\mathrm{M}, q}+C_{\mathrm{M}, \dot{\alpha}}\right)\right. \\
& \left.+2 \mu\left(C_{\mathrm{L}, q}+C_{\mathrm{L}, \dot{\alpha}}\right)\left(x_{\mathrm{cg}}-x_{\mathrm{m}}\right)\right), \\
& c=4 \mu^{2} C_{\mathrm{L}, \alpha}\left(x_{\mathrm{cg}}-x_{\mathrm{m}}^{\prime}\right)\left(1+O\left(10^{-3}\right)\right), \\
& d=-C_{\mathrm{L}}\left(\left(C_{\mathrm{M}, q}+C_{\mathrm{M}, \dot{\alpha}}\right)\left(2 C_{\mathrm{L}}+C_{\mathrm{L}, u}\right)+\gamma C_{\mathrm{L}, \alpha} C_{\mathrm{M}, q}+O\left(10^{-1}\right)\right) \\
& +2 C_{\mathrm{L}}\left(x_{\mathrm{cg}}-x_{\mathrm{s}}\right)\left(\left(C_{\mathrm{L}, q}+C_{\mathrm{L}, \dot{\alpha}}\right) C_{\mathrm{L}}+2 \mu C_{\mathrm{D}, \alpha}+O(1)\right) \\
& -2 \mu\left(x_{\mathrm{cg}}-x_{\mathrm{m}}\right) \\
& \times\left(C_{\mathrm{L}, \alpha}\left(C_{\mathrm{T}, u}-C_{\mathrm{D}, u}+3 \gamma C_{\mathrm{L}}\right)+2 C_{\mathrm{D}, \alpha} C_{\mathrm{L}}+O\left(10^{-2}\right)\right) \\
& +O\left(10^{-2}\right) \text {, } \\
& e=2 C_{\mathrm{L}}^{2} C_{\mathrm{L}, \alpha}\left(x_{\mathrm{cg}}-x_{\mathrm{s}}^{\prime}\right)\left(1+O\left(10^{-2}\right)\right),
\end{aligned}
$$

where

$$
\begin{aligned}
& x_{\mathrm{m}}^{\prime}=x_{\mathrm{m}}+\left(x_{\mathrm{m}}-x_{\mathrm{n}}\right) O\left(10^{-2} i_{y} / \mu\right), \\
& x_{\mathrm{s}}^{\prime}=x_{\mathrm{s}}+\left(x_{\mathrm{s}}-x_{\mathrm{n}}\right) O\left(10^{-2}\right) .
\end{aligned}
$$

Since $x_{\mathrm{m}}-x_{\mathrm{n}}$ and $x_{\mathrm{s}}-x_{\mathrm{n}}$ are of the order $10^{-1}$ by (8)-(10), therefore $x_{\mathrm{s}}^{\prime}=x_{\mathrm{s}}+O\left(10^{-3}\right)$ and $x_{\mathrm{m}}^{\prime}=x_{\mathrm{m}}+O\left(10^{-3} i_{y} / \mu\right)$. Full expression for $x_{s}^{\prime}$ can be found in (A.7)-(A.9).

It can be verified by direct substitution that the ratio $b / a$ is of the order $10^{-1}$, whereas the ratios $c / a, d / a$, and $e / a$ are of the orders $10\left(x_{\mathrm{cg}}-x_{\mathrm{m}}^{\prime}\right) i_{y}^{-1}, 10^{-3} i_{y}^{-1}$, and $10^{-3} i_{y}^{-1}\left(x_{\mathrm{cg}}-x_{\mathrm{s}}^{\prime}\right)$, respectively. Hence, as long as

$$
x_{\mathrm{cg}}-x_{\mathrm{m}} \text { is of the order } 10^{-1},
$$

the third and the fourth terms in the Routh sequence, $f=c-$ $a d / b$ and $g=d-b e / f$, can be approximated with $c$ and $d-b e / c$; the error of these approximations should be of the 
order of $10^{-2}$ relative to the respective leading terms. Explicit expression for $c$ is found in (12); explicit (approximate) expression for $g$ follows from (8) and (11)-(16). It can be regrouped in various forms; the following two,

$$
g=C_{\mathrm{L}, \alpha} C_{\mathrm{L}}^{2}\left(i_{2} \bar{X}+\left(i_{1}+i_{3}-\bar{i}_{y}\right)+\left(i_{0}-i_{3}+\bar{i}_{y}\right) \bar{X}^{-1}\right)
$$

and

$$
\begin{aligned}
g= & C_{\mathrm{L}, \alpha} C_{\mathrm{L}}^{2} \frac{\bar{X}-1}{\bar{X}} \\
& \times\left(\frac{i_{2}(\bar{X}-1)^{2}+\left(2 i_{2}+i_{1}+i_{3}\right)(\bar{X}-1)+\left(i_{0}+i_{1}+i_{2}\right)}{\bar{X}-1}-\bar{i}_{y}\right),
\end{aligned}
$$

will serve the needs of the following discussion. Here,

$$
\begin{aligned}
& \bar{X}=\frac{x_{\mathrm{cg}}-x_{\mathrm{m}}}{x_{\mathrm{s}}-x_{\mathrm{m}}} \\
& i_{0}=4 \mu k_{q} \frac{x_{\mathrm{n}}-x_{\mathrm{m}}}{C_{\mathrm{L}, \alpha}}=-\frac{2 k_{q} C_{\mathrm{M}, q}}{C_{L, \alpha}} \\
& i_{1}=\frac{2 \mu}{C_{\mathrm{L}, \alpha} C_{\mathrm{L}}}\left(\left(k_{q} C_{\mathrm{L}, u}+\gamma C_{\mathrm{L}, \alpha}\right)\left(x_{\mathrm{n}}-x_{\mathrm{m}}\right)-2 C_{\mathrm{D}, \alpha}\left(x_{\mathrm{s}}-x_{\mathrm{m}}\right)\right), \\
& i_{2}=\frac{2 \mu}{C_{\mathrm{L}}^{2}}\left(C_{\mathrm{D}, u}-C_{\mathrm{T}, u}-3 \gamma C_{\mathrm{L}}\right)\left(x_{\mathrm{s}}-x_{\mathrm{m}}\right), \\
& i_{3}=i_{0} \frac{C_{\mathrm{L}, q}}{2 \mu}=-\frac{k_{q} C_{\mathrm{M}, q} C_{\mathrm{L}, q}}{\mu C_{\mathrm{L}, \alpha}}, \\
& \bar{i}_{y}=\frac{i_{y}}{\mu},
\end{aligned}
$$

and in which

$$
k_{q}=1+\frac{C_{\mathrm{M}, \dot{\alpha}}}{C_{\mathrm{M}, q}}
$$

By the Routh criterion, the number of roots in the righthalf-plane equals the number of the sign alternations in $S$. Since, invariably, $C_{\mathrm{M}, q}<0, C_{\mathrm{M}, \dot{\alpha}}<0$, and $C_{\mathrm{L}, \alpha}>0$, the first two members of $S, a$, and $b$, are positive by (5), (11), (10) and (17). Hence, the aircraft is stable only if the remaining members, $f, g$, and $e$, are positive as well. The first of these conditions yields $x_{\mathrm{cg}}>x_{\mathrm{m}}^{\prime}$ (see (12)), or, approximately,

$$
x_{\mathrm{cg}}>x_{\mathrm{m}}
$$

In view of (17) this condition is almost redundant. We shall assume that it is satisfied unconditionally. The second of these conditions,

$$
g>0
$$

will be discussed in the following sections. The last of these conditions yields $x_{\mathrm{cg}}>x_{\mathrm{s}}^{\prime}($ see (14)), or, approximately,

$$
x_{\mathrm{cg}}>x_{\mathrm{s}} .
$$

It suggests that the center of gravity should be placed forward of the neutral speed stability point. There is nothing new in this result [1, page 335], but it is satisfying to obtain it here. In the analysis of (26b) we shall assume that this condition is satisfied as well. In conjunction with (26a) and (19) it implies that either $\bar{X}>1$ or $\bar{X}<0$.

Note that failing (26b), but not (26a) and (26c), implies two sign alternations in the Routh sequence and hence two divergent (phugoid) roots; failing (26c) only, or both (26c) and (26b), implies one divergent root.

\section{Condition (26b)}

The consequences of (26b) can be inferred from either (18a) or (18b), depending on the variable that is considered given. For example, the conjunction of (26b) and (18b) sets an upper limit on $\bar{i}_{y}$,

$$
\bar{i}_{y}<\bar{J}(\bar{X})
$$

where

$$
\bar{J}(\bar{X})=i_{2}(\bar{X}-1)+\left(2 i_{2}+i_{1}+i_{3}\right)+\frac{i_{0}+i_{2}+i_{1}}{\bar{X}-1} .
$$

Conversely, given $\bar{i}_{y}$, the conjunction of (26b) and (18a) sets limits on $\bar{X}$ :

$$
\begin{aligned}
& \text { if } i_{2}>0 \text { and } \bar{i}_{y} \geq \bar{J}_{0} \text {, then } \bar{X}_{-}\left(\bar{i}_{y}\right)<\bar{X}<0 \text { or } \\
& 0<\bar{X}<\bar{X}_{-}\left(\bar{i}_{y}\right) \text { or } 0<\bar{X}_{+}\left(\bar{i}_{y}\right)<\bar{X} \text {; }
\end{aligned}
$$

if $i_{2}>0$ and $\bar{i}_{y}<\bar{J}_{0}$, then $0<\bar{X}$;

$$
\text { if } i_{2}<0 \text {, then } \bar{X}<\bar{X}_{+}\left(\bar{i}_{y}\right)<0 \text { or } 0<\bar{X}<\bar{X}_{-}\left(\bar{i}_{y}\right) \text {. }
$$

Here,

$\bar{X}_{ \pm}\left(\bar{i}_{y}\right)=\frac{-\left(i_{1}+i_{3}-\bar{i}_{y}\right) \pm \sqrt{\left(i_{1}+i_{3}-\bar{i}_{y}\right)^{2}-4 i_{2}\left(i_{0}-i_{3}+\bar{i}_{y}\right)}}{2 i_{2}}$

are the values of $\bar{X}$ for which $g=0$, whereas

$$
\bar{J}_{0}=i_{1}+i_{3}+2 i_{2}+2 \sqrt{i_{2}\left(i_{0}+i_{1}+i_{2}\right)}
$$

is the value of $\bar{i}_{y}$ for which the square root in (30) vanishes with $i_{2}>0$. Note that $\bar{J}_{0}=\min _{\bar{X}>1} \bar{J}(\bar{X})$, by $(28)$.

\section{An Example}

The use of (27) and (29) can be elucidated on a practical example. Consider a fictitious long-endurance UAV. For this aircraft we adopt a standard drag-lift relation

$$
C_{\mathrm{D}}=C_{\mathrm{D} 0}+K C_{\mathrm{L}}^{2} ;
$$


neglect $C_{\mathrm{D}, u}, C_{\mathrm{L}, u}$, and $C_{\mathrm{M}, u}$; and set

$$
C_{\mathrm{T}, u}=-k_{\mathrm{T}}\left(C_{\mathrm{D}}+\gamma C_{\mathrm{L}}\right),
$$

where $k_{\mathrm{T}}$ is the scaling exponent of the propulsion system. It equals 2 for an ideal (constant thrust) jet and 3 for an ideal (constant power) propeller in [1, page 263]. Values of $C_{\mathrm{D} 0}, K$, $\mu$, and the relevant stability derivatives are listed in Table 1.

Using this data, equation (4) was solved numerically with many combinations of lift coefficient, stability margin $\left(C_{\mathrm{M}, \alpha}\right)$, and reduced moment of inertia. Differentiating the stable and unstable combinations yielded the stability boundaries drawn by the thick lines in Figure 1. Approximate boundaries have been constructed as contours $\bar{J}(\bar{X})=\bar{i}_{y}$ (see (27)) using (28), (8), and (19)-(25); they appear as thin lines on the same figure. The fit between the two seems fair.

With no $u$-derivatives, $x_{\mathrm{s}}=x_{\mathrm{s}}^{\prime}=x_{\mathrm{n}}$, by (9) and (16), and since $C_{\mathrm{M}, q}<0$, therefore $x_{\mathrm{s}}-x_{\mathrm{m}}>0$, by (8). Thus, $\bar{X}>1$, by (19) and (26a). Moreover,

$$
\begin{gathered}
i_{1}=-\frac{C_{\mathrm{M}, q}}{C_{\mathrm{L}}}\left(\gamma-2 \frac{C_{\mathrm{D}, \alpha}}{C_{\mathrm{L}, \alpha}}\right), \\
i_{2}=-\frac{C_{\mathrm{M}, q}}{C_{\mathrm{L}}}\left(k_{\mathrm{T}} \frac{C_{\mathrm{D}}}{C_{\mathrm{L}}}+\left(k_{\mathrm{T}}-3\right) \gamma\right),
\end{gathered}
$$

by (21) and (22).

With $k_{\mathrm{T}}=2$ (an ideal jet), $i_{2}$ turns negative once $\gamma$ exceeds $2 C_{\mathrm{D}} / C_{\mathrm{L}}$. In this case $(29 \mathrm{c})$ applies, inferring that in a (sufficiently) steep climb, the aircraft turns unstable with $\bar{X}>\bar{X}_{-}\left(\bar{i}_{y}\right)$. This behavior is explicit in Figure 1(b). An exhaustive discussion of the stability of a climbing jet can be found on pages 329-346 in [1] and hence will not be repeated here.

With $k_{\mathrm{T}}=3$ (an ideal propeller), $i_{2}$ is positive unconditionally. In this case, (27) applies, inferring that an idealpropeller-driven aircraft turns unstable for sufficiently large moment of inertia. This behavior is explicit in Figures 1(c) and $1(\mathrm{~d})$. Since $i_{0}, i_{2}$, and $i_{3}$ are independent of $\gamma$ by (20), (23), and (35) and $i_{1}$ increases with $\gamma$ by (34), it follows from $(28)$ that $\bar{J}(\bar{X})$ increases with $\gamma$ as well. Consequently, the lowest (and hence the most constricting) limit on the longitudinal moment of inertia of a propeller-driven aircraft is obtained in a power-off glide, where $\gamma=-C_{\mathrm{D}} / C_{\mathrm{L}}$. Indeed, the curves of constant $i_{y}$ in Figure 1(d) are lower than those in Figure 1(c).

In a glide,

$$
\begin{aligned}
\bar{J}(\bar{X})=-K C_{\mathrm{M}, q} & \left(\left(3+\frac{3 C_{\mathrm{D} 0}}{K C_{\mathrm{L}}^{2}}\right)(\bar{X}-1)\right. \\
& +\left(1+\frac{5 C_{\mathrm{D} 0}}{K C_{\mathrm{L}}^{2}}+\frac{2 k_{q}}{K C_{\mathrm{L}, \alpha}} \frac{C_{\mathrm{L}, q}}{2 \mu}\right) \\
& \left.+\left(\frac{2 k_{q}}{K C_{\mathrm{L}, \alpha}}-2+\frac{2 C_{\mathrm{D} 0}}{K C_{\mathrm{L}}^{2}}\right) \frac{1}{\bar{X}-1}\right),
\end{aligned}
$$

by (28), (32)-(35), (20), and (23). Since $\bar{X}>1$ (see above), $\bar{J}(\bar{X})$ decreases with increasing lift coefficient. Indeed, the lines of constant $i_{y}$ in Figure 1(d) are higher for lower values
TABLE 1: Dimensionless stability derivatives of the model aircraft.

\begin{tabular}{lccc}
\hline Quantity & Value & Quantity & Value \\
\hline$C_{\mathrm{L}}$ & variable & $C_{\mathrm{M}, u}$ & 0 \\
$C_{\mathrm{L}, \alpha}$ & 6.3 & $C_{\mathrm{D} 0}$ & 0.03 \\
$C_{\mathrm{L}, \dot{\alpha}}$ & 1 & $K$ & 0.03 \\
$C_{\mathrm{L}, q}$ & 4.5 & $C_{\mathrm{D}, \alpha}$ & $0.38 C_{\mathrm{L}}$ \\
$C_{\mathrm{L}, u}$ & 0 & $C_{\mathrm{D}, u}$ & 0 \\
$C_{\mathrm{M}, \alpha}$ & variable & $C_{\mathrm{T}, u}$ & variable \\
$C_{\mathrm{M}, \dot{\alpha}}$ & -4.0 & $\mu$ & 150 \\
$C_{\mathrm{M}, q}$ & -20.0 & $i_{y}$ & variable \\
\hline
\end{tabular}

of $i_{y}$. Accordingly, the most constricting limit on the moment of inertia in a glide is obtained when the aircraft flies at the highest possible lift coefficient.

\section{Maximal Moment of Inertia}

Let $\bar{J}_{1}=\min _{\left(\bar{X}>1, C_{\mathrm{L}}>0\right)} \bar{J}(\bar{X})$ be the minimum of $\bar{J}(\bar{X})$ in a glide with respect to both $\bar{X}$ and $C_{\mathrm{L}}$. Under the same assumptions as those of the preceding section (the parabolic drag polar and the neglect of the $u$-derivatives) it is given by

$$
\bar{J}_{1}=-2 C_{\mathrm{M}, q} P
$$

where

$$
P=K\left(\frac{1}{2}+\frac{k_{q}}{K C_{\mathrm{L}, \alpha}} \frac{C_{\mathrm{L}, q}}{2 \mu}+\sqrt{\frac{6 k_{q}}{K C_{\mathrm{L}, \alpha}}-6}\right) .
$$

This minimum corresponds to $C_{\mathrm{L}} \rightarrow \infty$ (which is, of course, beyond reach) and

$$
\bar{X}=1+\sqrt{\frac{2 k_{q} /\left(K C_{\mathrm{L}, \alpha}\right)-2+2 C_{\mathrm{D} 0} /\left(K C_{\mathrm{L}}^{2}\right)}{3+3 C_{\mathrm{D} 0} /\left(K C_{\mathrm{L}}^{2}\right)}} .
$$

Clearly, an aircraft which is designed with sufficiently small moment of inertia to satisfy $\bar{i}_{y}<\bar{J}_{1}$ and with sufficiently forward center-of-gravity to satisfy (26c) (and (26a)) will be stable at all flight conditions.

It is shown in Appendix B that $P$ depends mainly on the aspect ratio of the wing. Since the aspect ratio is dictated by performance (rather than stability) requirements, $C_{\mathrm{M}, q}$ remains the main design parameter affecting the maximal moment of inertia for which the aircraft is stable.

For a conventional subsonic aircraft, $C_{\mathrm{M}, q}$ is associated mainly with the contribution of its horizontal tail; the contribution of the other parts is small and has the same (negative) sign as the tail. Hence, the contribution of the tail,

$$
C_{\mathrm{M}, q}=-2 a_{\mathrm{t}}\left(x_{\mathrm{n}}-x_{\mathrm{t}}\right)^{2}\left(\frac{S_{\mathrm{t}}}{S_{\mathrm{w}}}\right)
$$

provides a lower bound on $\left|C_{\mathrm{M}, q}\right|$. Here, $S_{\mathrm{t}} / S_{\mathrm{w}}$ is the tail to wing areas ratio, $x_{\mathrm{n}}-x_{\mathrm{t}}$ is the distance between the aircraft neutral point and the aerodynamic center of the tail, and $a_{\mathrm{t}}$ is the lift slope coefficient of the tail [1, page 269]. 


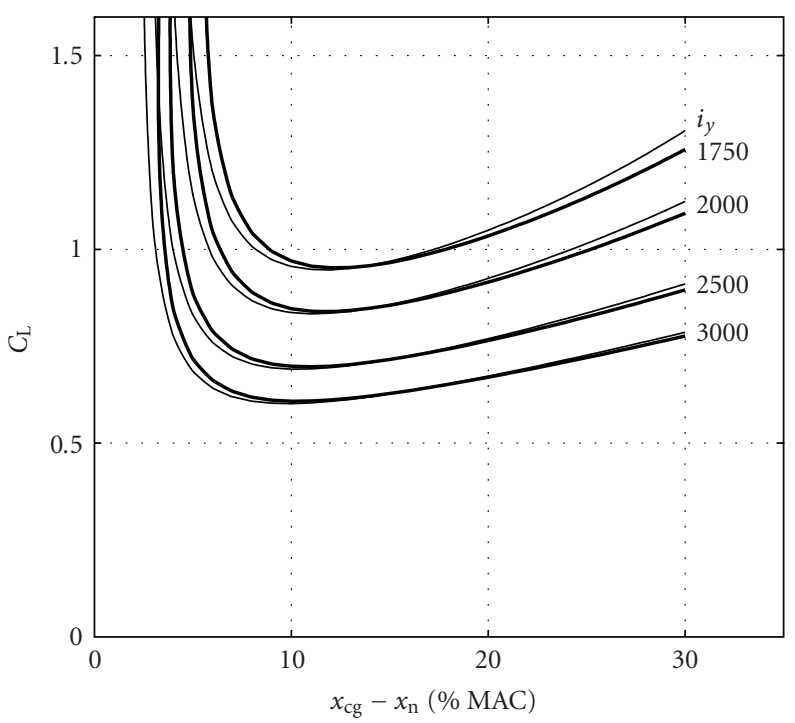

(a) level flight, $k_{\mathrm{T}}=2$

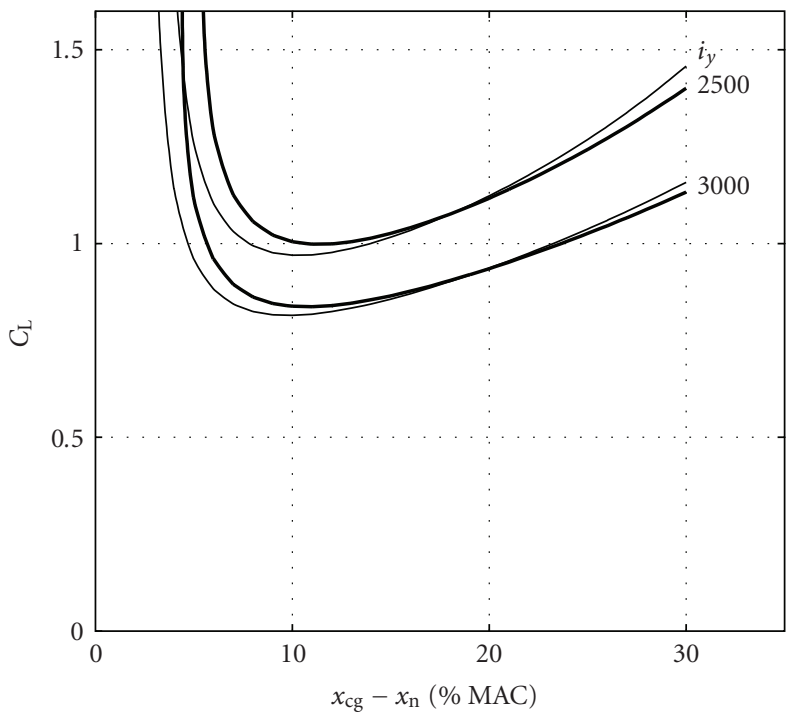

(c) level flight, $k_{\mathrm{T}}=3$

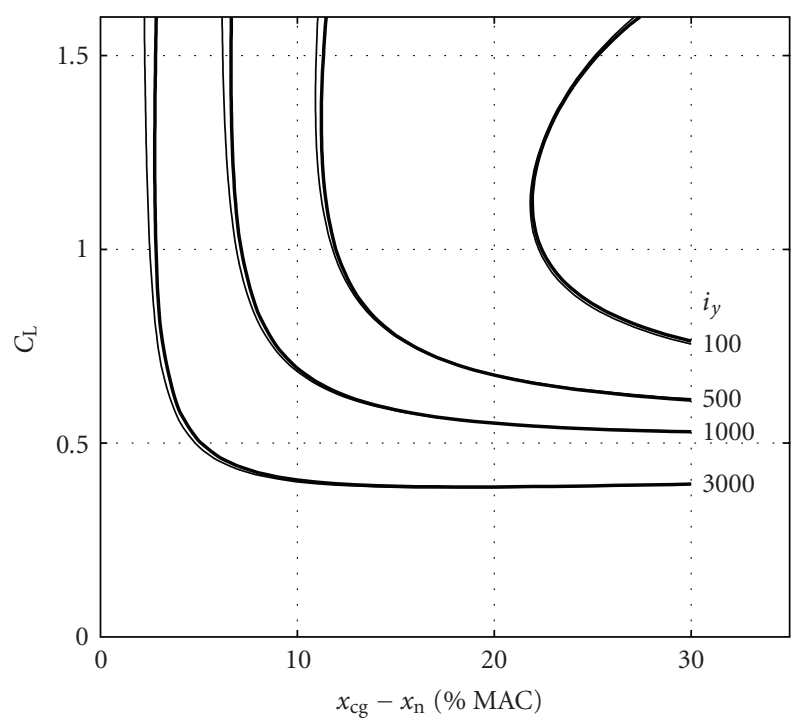

(b) 8 deg climb, $k_{\mathrm{T}}=2$

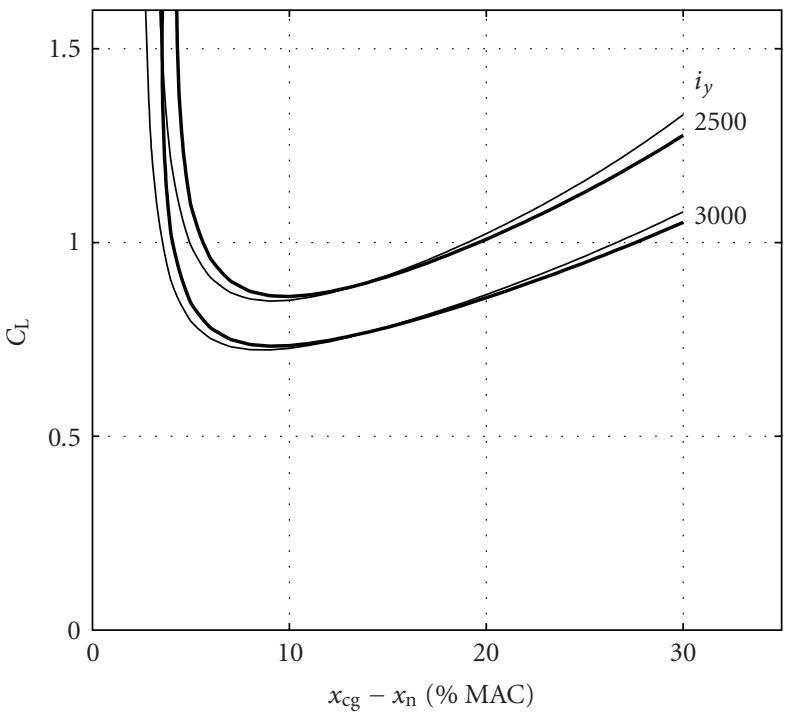

(d) power off glide

Figure 1: Stability boundaries for the model aircraft. Combinations of lift coefficient and stability margin are unstable if situated to the right (b) or above $(\mathrm{a}, \mathrm{c}, \mathrm{d})$ the respective line. Thick lines mark the exact boundaries obtained from multiple solutions of (4); thin lines are solutions of $\bar{J}(\bar{X})=\bar{i}_{y}$.

$\bar{i}_{y}$ can be related with longitudinal (reduced) radius of gyration, $r_{y}$. In fact, $\bar{i}_{y}=4 r_{y}^{2}$ (see the Nomenclature). Hence, one may set $\bar{J}_{1}=4 R_{1}^{2}$ and use $R_{1}$ as a conservative upper bound of $r_{y}$;

$$
R_{1}=\left(x_{\mathrm{n}}-x_{\mathrm{t}}\right) \sqrt{\frac{a_{\mathrm{t}} P S_{\mathrm{t}}}{S_{\mathrm{w}}}}
$$

by (37), (38), and (40). With $a_{\mathrm{t}}=5, S_{\mathrm{t}} / S_{\mathrm{w}}=6$, and the data of our model aircraft, $R_{1}$ equals, approximately, $0.4\left(x_{\mathrm{n}}-x_{\mathrm{t}}\right)$ almost half the distance between the tail and the center-ofgravity (it is implied here that $\left.\left(x_{\mathrm{cg}}-x_{\mathrm{n}}\right) \ll\left(x_{\mathrm{n}}-x_{\mathrm{t}}\right)\right)$. This result marks out a single configuration which may be at risk of becoming unstable because of the excessive inertia. This is the configuration with an aft engine, counterbalanced by the equipment in the nose. All conventional configurations of propeller-driven aircraft should be stable as long as their center-of-gravity is properly placed.

\section{Concluding Remarks}

The key element underlying the preceding derivations is that, under assumptions (10) and (17), the coefficients $a, \ldots, e$ of the characteristic polynomial can be approximated by a few dominant terms only. These dominant terms were used in (11)-(14) and (18) to approximate the respective members in the Routh sequence, leading to stability conditions (26b) and (26c). The former sets an upper limit on the longitudinal 
moment of inertia; the latter sets an aft limit on the centerof-gravity position.

Perhaps the most conspicuous result of this study is that most configurations of propeller-driven aircraft are practically unlimited by inertia and hence are stable as long as their center-of-gravity is placed forward of the neutralspeed-stability (and maneuver) points-condition (26c). At the same time, aircraft with high-aspect ratio wing and an aft engine are at risk of becoming unstable because of an excessive inertia-condition (26b). The critical moment of inertia (28) depends on the center-of-gravity position and the flight conditions, but $\bar{J}_{1}$ from (37) may provide an early warning when longitudinal inertia becomes an issue.

\section{Appendices}

\section{A. Coefficients of the Characteristic Polynomial}

The coefficients in (4) are

$$
\begin{aligned}
& a=2 i_{y} \mu\left(2 \mu+C_{\mathrm{L}, \dot{\alpha}}\right), \\
& b=2 \mu\left(-2 \mu\left(C_{\mathrm{m}, q}+C_{\mathrm{m}, \dot{\alpha}}\right)+i_{y}\left(C_{\mathrm{L}, \alpha}+C_{\mathrm{D}}\right)\right. \\
& \left.+\left(C_{\mathrm{m}, \dot{\alpha}} C_{\mathrm{L}, q}-C_{\mathrm{L}, \dot{\alpha}} C_{\mathrm{m}, q}\right)\right) \\
& -i_{y}\left(2 \mu+C_{\mathrm{L}, \dot{\alpha}}\right)\left(C_{\mathrm{T}, u}-C_{\mathrm{D}, u}+2 \gamma C_{\mathrm{L}}\right), \\
& c=2 \mu\left(-\left(2 \mu-C_{L, q}\right) C_{\mathrm{m}, \alpha}-C_{\mathrm{m}, q}\left(C_{\mathrm{L}, \alpha}+C_{\mathrm{D}}+\gamma C_{\mathrm{L}}\right)\right. \\
& \left.+\left(C_{\mathrm{T}, u}-C_{\mathrm{D}, u}+3 \gamma C_{\mathrm{L}}\right)\left(C_{\mathrm{m}, q}+C_{\mathrm{m}, \dot{\alpha}}\right)\right) \\
& +i_{y}\left(\left(2 C_{\mathrm{L}}+C_{\mathrm{L}, u}\right)\left(C_{\mathrm{L}}-C_{\mathrm{D}, \alpha}\right)\right. \\
& \left.-\left(C_{\mathrm{L}, \alpha}+C_{\mathrm{D}}\right)\left(C_{\mathrm{T}, u}-C_{\mathrm{D}, u}+2 \gamma C_{\mathrm{L}}\right)\right) \\
& -\left(C_{\mathrm{T}, u}-C_{\mathrm{D}, u}+2 \gamma C_{\mathrm{L}}\right)\left(C_{\mathrm{m}, \dot{\alpha}} C_{\mathrm{L}, q}-C_{\mathrm{m}, q} C_{\mathrm{L}, \dot{\alpha}}\right), \\
& d=-\left(2 C_{\mathrm{L}}+C_{\mathrm{L}, u}\right)\left(C_{\mathrm{L}}\left(C_{\mathrm{m}, q}+C_{\mathrm{m}, \dot{\alpha}}\right)-C_{\mathrm{m}, q} C_{\mathrm{D}, \alpha}\right) \\
& +\left(C_{\mathrm{T}, u}-C_{\mathrm{D}, u}+2 \gamma C_{\mathrm{L}}\right) \\
& \times\left(-\gamma C_{\mathrm{L}} C_{\mathrm{m}, \dot{\alpha}}+\left(C_{\mathrm{D}}+C_{\mathrm{L}, \alpha}\right) C_{\mathrm{m}, q}+\left(2 \mu-C_{\mathrm{L}, q}\right) C_{\mathrm{m}, \alpha}\right) \\
& +C_{\mathrm{m}, u}\left(C_{\mathrm{L}}\left(C_{\mathrm{L}, q}+C_{\mathrm{L}, \dot{\alpha}}\right)+\left(2 \mu-C_{\mathrm{L}, q}\right) C_{\mathrm{D}, \alpha}\right) \\
& +2 \mu C_{\mathrm{L}} \gamma C_{\mathrm{m}, \alpha}, \\
& e=-C_{\mathrm{m}, \alpha} C_{\mathrm{L}}\left(2 C_{\mathrm{L}}+C_{\mathrm{L}, u}+\left(C_{\mathrm{T}, u}-C_{\mathrm{D}, u}+2 C_{\mathrm{L}} \gamma\right) \gamma\right) \\
& +C_{\mathrm{m}, u} C_{\mathrm{L}}\left(C_{\mathrm{L}, \alpha}+C_{\mathrm{D}}+\left(C_{\mathrm{L}}-C_{\mathrm{D}, \alpha}\right) \gamma\right) .
\end{aligned}
$$
as

Using (6), (7), and (9), the last expression can be recast

$$
\begin{aligned}
e & =2 C_{\mathrm{L}}^{2} C_{\mathrm{L}, \alpha}\left(x_{\mathrm{cg}}-x_{\mathrm{s}}^{\prime}\right) \\
& \times\left(1-\frac{C_{\mathrm{D}} C_{\mathrm{L}, u}}{2 C_{\mathrm{L}} C_{\mathrm{L}, \alpha}}+\gamma\left(\gamma-\frac{C_{\mathrm{L}, u}\left(C_{\mathrm{L}}-C_{\mathrm{D}, \alpha}\right)}{2 C_{\mathrm{L}} C_{\mathrm{L}, \alpha}}+\frac{C_{\mathrm{T}, u}-C_{\mathrm{D}, u}}{2 C_{\mathrm{L}}}\right)\right),
\end{aligned}
$$

where

$$
x_{\mathrm{s}}^{\prime}=x_{n}-\frac{C_{\mathrm{M}, u}}{2 C_{\mathrm{L}}} \frac{C_{1}}{C_{2}}
$$

and in which

$$
\begin{aligned}
C_{1}= & 1-\frac{C_{\mathrm{D}}\left(C_{\mathrm{L}}+C_{\mathrm{L}, u}\right)}{C_{\mathrm{L}} C_{\mathrm{L}, \alpha}} \\
& +2 \gamma\left(\gamma-\frac{\left(C_{\mathrm{L}}-C_{\mathrm{D}, \alpha}\right)\left(C_{\mathrm{L}}+C_{\mathrm{L}, u}\right)}{2 C_{\mathrm{L}} C_{\mathrm{L}, \alpha}}+\frac{\left(C_{\mathrm{T}, u}-C_{\mathrm{D}, u}\right)}{2 C_{\mathrm{L}}}\right),
\end{aligned}
$$

$$
\begin{aligned}
C_{2}= & 1-\frac{C_{\mathrm{D}} C_{\mathrm{L}, u}}{2 C_{\mathrm{L}} C_{\mathrm{L}, \alpha}} \\
& +\gamma\left(\gamma-\frac{\left(C_{\mathrm{L}}-C_{\mathrm{D}, \alpha}\right) C_{\mathrm{L}, u}}{2 C_{\mathrm{L}} C_{\mathrm{L}, \alpha}}+\frac{\left(C_{\mathrm{T}, u}-C_{\mathrm{D}, u}\right)}{2 C_{\mathrm{L}}}\right)
\end{aligned}
$$

\section{B. Parameter $P$}

In this appendix we seek the leading-order approximation for $P$, which was defined in (38). It depends on four parameters: $k_{q}, C_{\mathrm{L}, \alpha}, K$, and the ratio $C_{\mathrm{L}, q} / \mu$. The former is given by (25). Neglecting the respective contributions of the horizontal tail, and assuming that the wing loading is elliptic, the simplest approximations for the next two are

$$
\begin{aligned}
C_{\mathrm{L}, \alpha} & =\frac{2 \pi A_{\mathrm{w}}}{A_{\mathrm{w}}+2}, \\
K & =\frac{1}{\pi A_{\mathrm{w}}},
\end{aligned}
$$

where $A_{\mathrm{w}}$ is the aspect ratio of the wing [4, page 130]. With these,

$$
\begin{aligned}
P= & \frac{C_{\mathrm{L}, q}}{4 \pi \mu}\left(1+\frac{C_{\mathrm{M}, \dot{\alpha}}}{C_{\mathrm{M}, q}}\right) \\
& +\frac{1}{\pi} \sqrt{\frac{3}{A_{\mathrm{W}}}+\frac{3}{A_{\mathrm{w}}} \frac{C_{\mathrm{M}, \dot{\alpha}}}{C_{\mathrm{M}, q}}+\frac{6}{A_{\mathrm{w}}^{2}} \frac{C_{\mathrm{M}, \dot{\alpha}}}{C_{\mathrm{M}, q}}} \\
& +\frac{1}{2 \pi A_{\mathrm{W}}}\left(1+\frac{C_{\mathrm{L}, q}}{\mu}+\frac{C_{\mathrm{L}, q}}{\mu} \frac{C_{\mathrm{M}, \dot{\alpha}}}{C_{\mathrm{M}, q}}\right) .
\end{aligned}
$$

The ratio $C_{\mathrm{M}, \dot{\alpha}} / C_{\mathrm{M}, q}$ is, approximately, the downwash derivative at the tail $[1, \mathrm{pp} .269,284]$, about 0.3 . The ratio $C_{\mathrm{L}, q} / \mu$ for a conventional subsonic aircraft (ultralights excluded) is of the order of $10^{-1}$ - see (10) and (10); the aspect ratio $A_{\mathrm{W}}$ is of the order of $10^{1}$. Hence, the leadingorder $\left(10^{-1}\right)$ term in $(\mathrm{B} .3)$ is $\pi^{-1} \sqrt{3 A_{w}^{-1}}$. 


\section{Nomenclature}

$a_{\mathrm{t}}$ : Lift slope coefficient of the horizontal tail

$a, \ldots, e: \quad$ Coefficients in the characteristic equation

$A_{\mathrm{w}}$ : $\quad$ Aspect ratio of the wing

$c_{\mathrm{W}}$ : $\quad$ Mean aerodynamic chord of the wing

$C_{\mathrm{D}}, C_{\mathrm{L}}, C_{\mathrm{T}}$ : Drag, lift, and thrust coefficients at trim; the unit is $(1 / 2) \rho V^{2} S_{\mathrm{W}}$

$C_{\mathrm{D} 0}$ : $\quad$ Parasite (zero lift) drag coefficient

$C_{\mathrm{m}}, C_{\mathrm{M}}$ : Pitching moment coefficients; the former is referred to the center of gravity, the latter is referred to the neutral point; the unit is $(1 / 2) \rho V^{2} S_{\mathrm{w}} c_{\mathrm{w}}$

$f, g: \quad$ The third and the fourth terms in the Routh sequence

$i_{0}, \ldots, i_{3}$ : Combinations of stability derivatives

$i_{y}, \bar{i}_{y}: \quad$ Reduced moment of inertia; the units are $(1 / 8) \rho S_{\mathrm{w}} c_{\mathrm{w}}^{3}$ and $(1 / 8) \rho S_{\mathrm{w}} c_{\mathrm{w}}^{3} \mu$

$\bar{J}(\bar{X}): \quad$ Critical value of $\bar{i}_{y}$ for a given $\bar{X}$

$\bar{J}_{0}, \bar{J}_{1}: \quad \min _{\bar{X}>1} \bar{J}(\bar{X})$ and $\min _{\left(\bar{X}>1, C_{L}>0\right)} \bar{J}(\bar{X})$, respectively

$k_{q}$ : $\quad$ Combination of the pitch damping derivatives defined in (25)

$k_{\mathrm{T}}$ : $\quad$ Scaling exponent of the thrust coefficient variation with airspeed at constant throttle

$K: \quad$ Induced drag coefficient

$P: \quad$ Parameter defined in (38)

q: $\quad$ Reduced pitch rate, $\dot{\theta}$

$r_{y}: \quad \quad$ Reduced radius of gyration, $(1 / 2) \sqrt{\bar{i}_{y}}$;

the unit is $c_{\mathrm{W}}$

$R_{1}$ : $\quad$ An upper stability limit for $r_{y}$

$S_{\mathrm{w}}, S_{\mathrm{t}}$ : Wing and tail areas

$t$ : $\quad$ Reduced time; the unit is $c_{\mathrm{W}} /(2 \mathrm{~V})$

$u$ : $\quad$ Reduced airspeed perturbation from trim; the unit is $V$

$V: \quad$ Airspeed at trim

$x_{\mathrm{cg}}$ : $\quad$ Reduced longitudinal center-of-gravity position; the unit is $\mathcal{c}_{\mathrm{W}}$

$x_{\mathrm{m}}, x_{\mathrm{n}}, x_{\mathrm{s}}$ : Maneuver, neutral, and neutral-speed-stability points; the unit is $c_{\mathrm{W}}$

$\bar{X}: \quad$ Reduced maneuver margin; the unit is $x_{\mathrm{s}}-x_{\mathrm{m}}$

$\bar{X}_{ \pm}\left(\bar{i}_{y}\right): \quad$ Critical values of $\bar{X}$ for a given $\bar{i}_{y}$

$\alpha: \quad$ Angle of attack, measured from trim

$\gamma: \quad$ Flight path angle at trim

$\theta: \quad$ Pitch angle, measured from trim

$\mu: \quad$ Reduced mass; the unit is $(1 / 2) \rho S_{\mathrm{W}} c_{\mathrm{W}}$

$\rho: \quad$ Air density.

\section{Subscripts}

$\ldots, \bullet$ Partial derivative with respect to $\bullet$, computed at trim.

\section{References}

[1] B. Etkin, Dynamics of Atmospheric Flight, John Wiley \& Sons, New York, NY, USA, 1972.

[2] R. D. Milne and G. D. Padfield, "Strongly controlled aircraft," Aeron Quart, vol. 22, no. 2, pp. 146-168, 1971.

[3] K. Ogata, Modern Control Engineering, Prentice Hall, Upper Saddle River, NJ, USA, 1970.

[4] B. Thwaites, Incompressible Aerodynamics, Oxford University Press, Oxford, UK, 1960.

\section{Overheads}

$\therefore$.: (overdot) a derivative with respect to the reduced time.

... (overbar) a nonstandard reduced quantity. 

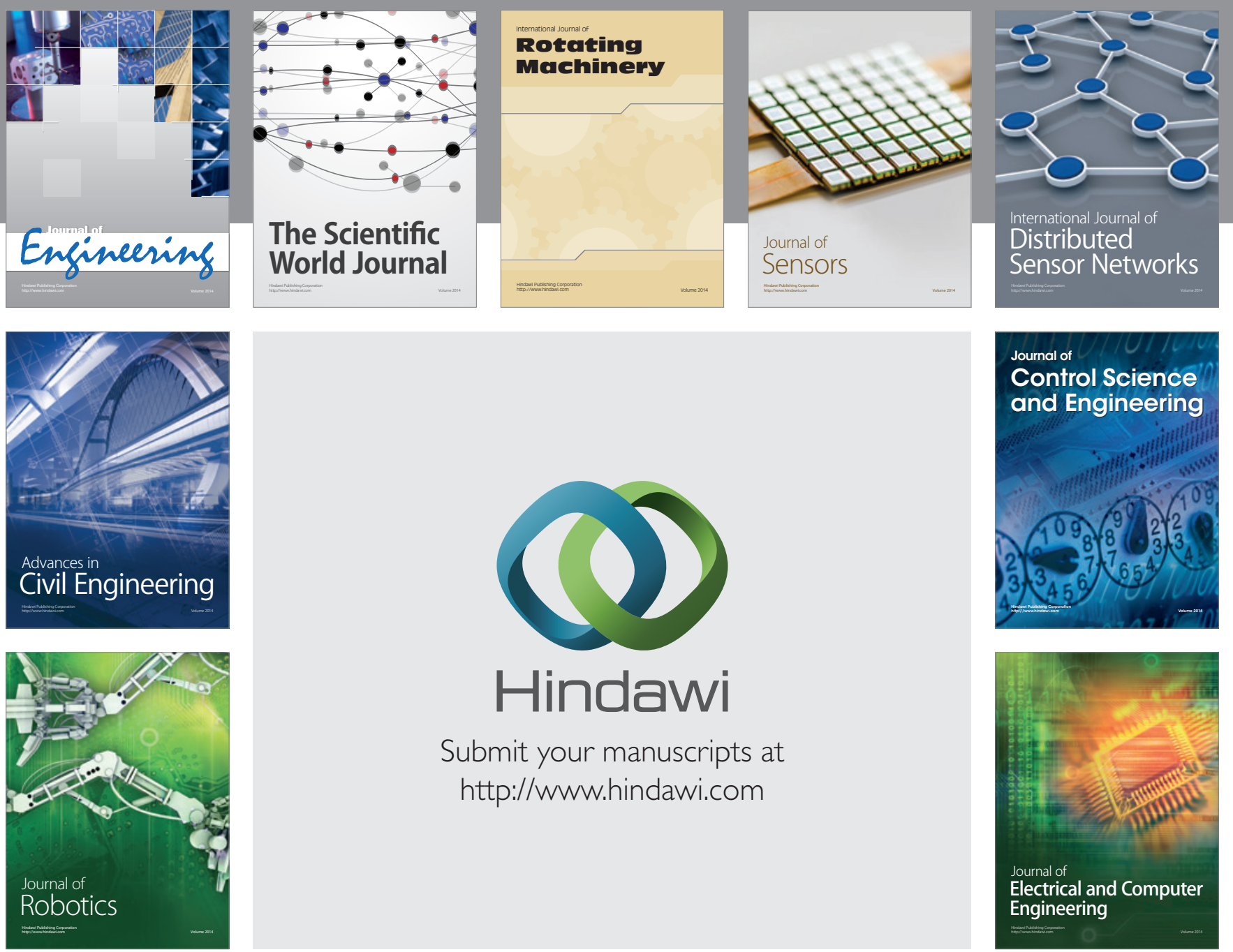

Submit your manuscripts at

http://www.hindawi.com
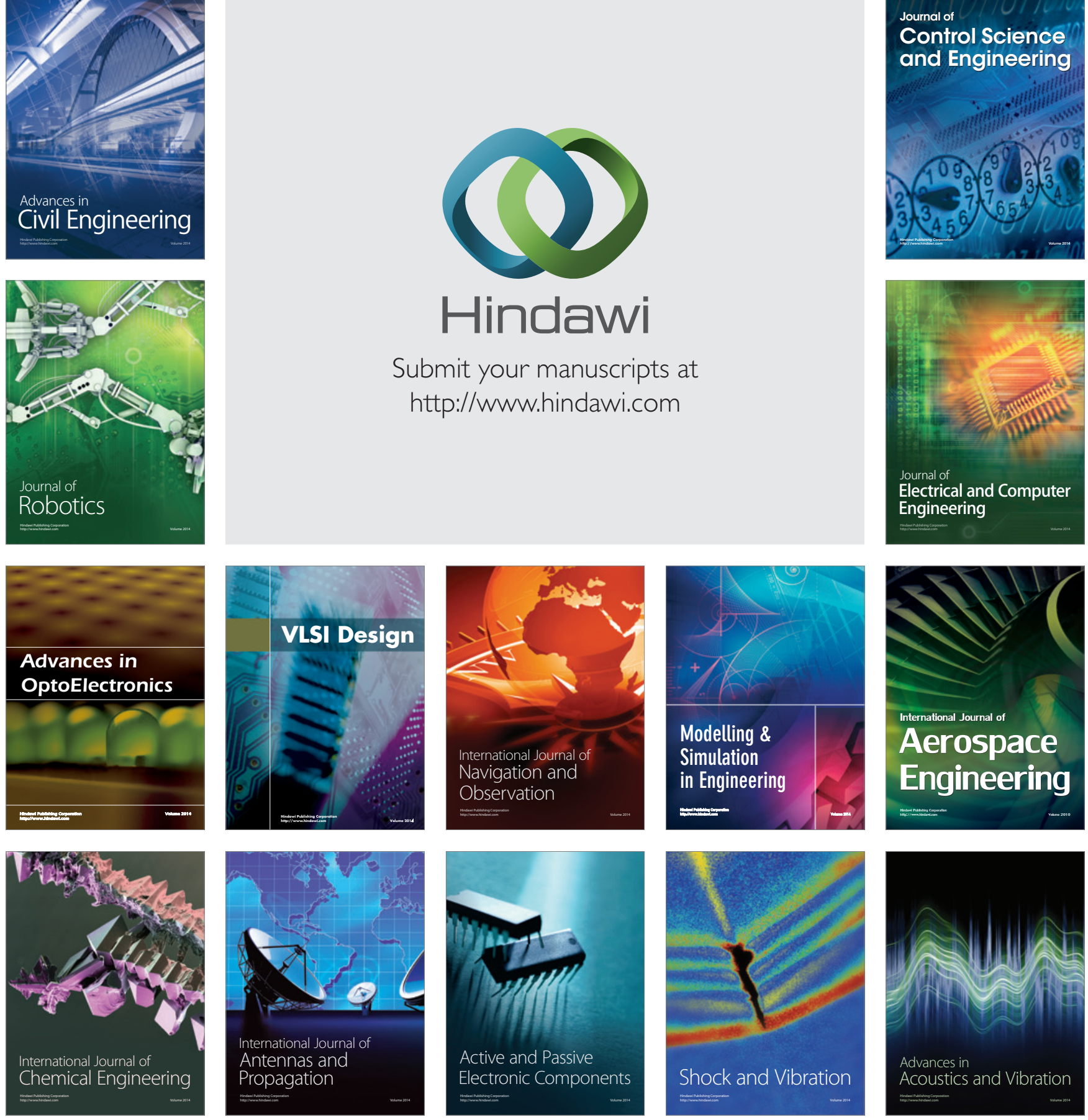